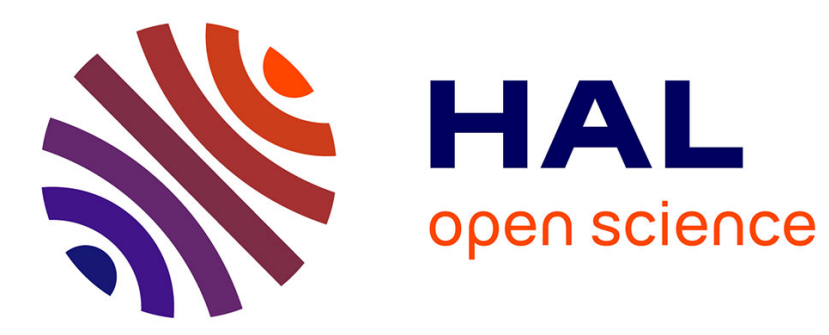

\title{
Micromechanical modeling of the linear viscoelasticity of carbon-black filled styrene butadiene rubbers: the role of the rubber-filler interphase
}

Julie Diani, Pierre Gilormini, Yannick Merckel, Fabien Vion-Loisel

\section{- To cite this version:}

Julie Diani, Pierre Gilormini, Yannick Merckel, Fabien Vion-Loisel. Micromechanical modeling of the linear viscoelasticity of carbon-black filled styrene butadiene rubbers: the role of the rubberfiller interphase. Mechanics of Materials, 2013, 59, pp.65-72. 10.1016/j.mechmat.2012.12.007 . hal00795101

\author{
HAL Id: hal-00795101 \\ https://hal.science/hal-00795101
}

Submitted on 28 Feb 2013

HAL is a multi-disciplinary open access archive for the deposit and dissemination of scientific research documents, whether they are published or not. The documents may come from teaching and research institutions in France or abroad, or from public or private research centers.
L'archive ouverte pluridisciplinaire HAL, est destinée au dépôt et à la diffusion de documents scientifiques de niveau recherche, publiés ou non, émanant des établissements d'enseignement et de recherche français ou étrangers, des laboratoires publics ou privés. 


\title{
Micromechanical modeling of the linear viscoelasticity of carbon-black filled styrene butadiene rubbers: the role of the rubber-filler interphase
}

\author{
Julie Diani ${ }^{\mathrm{a}, *}$, Pierre Gilormini ${ }^{\mathrm{a}}$, Yannick Merckel $^{\mathrm{b}}$, Fabien Vion-Loisel $^{\mathrm{c}}$ \\ ${ }^{a}$ PIMM, Arts et Métiers ParisTech, CNRS, 151 bd de l'Hôpital, 75013 Paris, France \\ ${ }^{b}$ Laboratoire LML, CNRS, Ecole Centrale de Lille, bd Paul Langevin, 59650 Villeneuve \\ d'Ascq, France \\ ${ }^{c}$ Manufacture Française des Pneumatiques Michelin, CERL Ladoux, 63040 Clermont \\ Ferrand, France
}

\begin{abstract}
Micromechanics modeling of the linear viscoelasticity of carbon-black filled styrene butadiene rubbers (SBR) shows that a simple representation of a spherical rigid-phase surrounded by rubber gum and embedded in an homogeneous equivalent medium provides access to the effective volume fraction of fillers. This simple representation is successful for a significant range of filler amount, and for materials in the glassy state. For materials in the rubbery state, experimental results support the existence of a filler-rubber interphase with reduced mobility due to confinement. The 4-phase micromechanics model, which accounts for a bounded rubber layer coating the fillers, provides satisfactory estimates of the linear viscoelasticity of filled rubbers from the rubbery state to the glassy state. It also provides access to the fillerrubber interphase behavior that appears viscoelastic, and to an estimate of the interphase thickness.
\end{abstract}

Keywords: filled rubbers, viscoelasticity, micromechanics modeling, interphase

\footnotetext{
${ }^{*}$ Corresponding author. Tel.: +33 144246192

Email address: julie.diani@ensam.eu (Julie Diani)
} 


\section{Introduction}

Few studies have attempted to calculate the linear viscoelasticity of carbonblack filled rubbers by a micromechanical approach and yet such an attempt could bring to light several aspects of the microstructure for these materials reinforced by nano-size particles, without adding difficulties encountered in nonlinear viscoelasticity such as the Payne effect. Moreover, with comparison to the carbon nanotubes for instance (Seidel and Lagoudas, 2005; Aldraihem, 2011), the spherical-like geometry of the carbon-black nano-particles is a good candidate to study the role of the filler-matrix interphase by applying a micromechanical approach since it provides analytical solutions. For instance, in the case of spherical geometries, the 3-phase model and the 4-phase model, accounting for spherical layers of materials of different behaviors, are easy to test. The interest of nano-particles is that they may introduce a size effect rendering mechanically effective the particle-matrix interphase. Among previous studies, one may cite the work of Albérola and co-workers (Albérola et al., 1997, 2001; Mélé et al., 2001), who based their composite microstructure representation on the cluster-cluster aggregation model (Heinrich and Klüppel, 2002). In their representation, the material is defined by a sphere of occluded rubber surrounded by a first layer of rigid filler and a second layer of unfilled rubber, and the whole layered sphere is embedded in the homogeneous equivalent medium (HEM), which is the composite. This modeling was shown to overestimate the experimental data in the rubbery state (Albérola et al., 1997; Mélé et al., 2001), and therefore suggested a misrepresentation of the material actual microstructure and phase interactions.

Actually, the former model accounts for an occluded phase increasing the effective volume fraction of rigid phase but does not account for the possible existence of a filler-rubber interphase with reduced mobility that was revealed recently by nuclear magnetic resonance spectroscopy in model silica filled rubbers (Berriot et al., 2002a) and carbon-black filled styrene butadiene rubbers (Léopoldès et al., 2004). According to Berriot et al. (2002b), at the filler-rubber interface, a layer of rubber bounded to the filler shows reduced mobility evidencing a behavior from glassy at the filler interface to rubbery as parting from the interface.

In this paper, testing the linear viscoelasticity of several materials made of the same rubber gum, characterized unfilled and filled with various amounts $5,30,40,50$ and 60 in parts per hundred rubber of the same carbon-black fillers, and using a micromechanics modeling approach, will provide informa- 
tion on the material microstructure and constitutive phases. The effective amount of fillers, the role of the filler-rubber interphase on the linear viscoelasticity of the composites, the behavior and the thickness of the interphase will be assessed by micromechanics modeling.

\section{Experiments}

\subsection{Material and tests}

Materials were manufactured by Michelin. They consist of carbon-black filled styrene butadiene rubbers (SBR). The rubber is a random copolymer with $15 \%$ mole fraction of styrene, with a molecular mass of $120 \mathrm{~g} / \mathrm{mol}$ and a density of $0.94 \mathrm{~g} / \mathrm{cm}^{3}$. The fillers are N347 carbon-black fillers, which fineness and structure are characterized by nitrogen adsorption using Brunner Emmet Teller analysis to $87 \pm 5 \mathrm{~m}^{2} / \mathrm{g}$ and by dibutyl-phtalate adsorption to $124 \pm 5 \mathrm{ml} / 100 \mathrm{~g}$ respectively. During the process, the fillers and the rubber are mixed with antioxidant (6PPD) and activators $(\mathrm{ZnO})$ in a thermal chamber, then curing agents (Sulfur and CBS) are added within a two-roll mill. Six materials, labeled according to the amount of fillers in parts per hundred rubber (phr), were manufactured: M0, M5, M30, M40, M50 and M60. Material compositions are listed in Table 1. According to the component densities, the corresponding volume fractions of fillers for these materials are $0,2.4 \%, 13.0 \%, 16.7 \%, 20.0 \%$ and $23.0 \%$ respectively. The material glass transition temperatures were obtained as the inflection temperatures measured by differential scanning calorimetry (DSC) using a TA instruments Q10 DSC at a heating rate of $10^{\circ} \mathrm{C} / \mathrm{min}$. Each material was then tested in dynamic mechanical analysis (DMA) using rectangular samples cut to the dimensions $2.5 \times 5.0 \times 30 \mathrm{~mm}^{3}$. DMA tests were run in tension on a TA Instruments Q800 analyzer. Samples were submitted to frequency sweeps at $0.1 \%$ strain for frequencies ranging from 0.01 to $10 \mathrm{~Hz}$. The amplitude of strain was chosen in order to remain in the linear viscoelastic strain range and to avoid any Payne effect. The temperature was increased stepwise from $-45^{\circ} \mathrm{C}$ to $-20^{\circ} \mathrm{C}$ with $5^{\circ} \mathrm{C}$ temperature increments.

\subsection{Results}

The DSC curves of the SBR samples are shown in Fig. 1. All samples showed a similar glass transition temperature of approximately $-48^{\circ} \mathrm{C}$. The presence of fillers did not affect the glass transition significantly. The DMA tests were used to characterize the linear viscoelasticity of each material. By 


\begin{tabular}{lcccccc}
\hline Material & M0 & M5 & M30 & M40 & M50 & M60 \\
Gum (SBR) & 100 & 100 & 100 & 100 & 100 & 100 \\
Carbon black (N347) & 0 & 5 & 30 & 40 & 50 & 60 \\
Accelerator (CBS) & 1.6 & 1.6 & 1.6 & 1.6 & 1.6 & 1.6 \\
Antioxidant (6PPD) & 1.9 & 1.9 & 1.9 & 1.9 & 1.9 & 1.9 \\
Stearic acid & 2.0 & 2.0 & 2.0 & 2.0 & 2.0 & 2.0 \\
Sulfur & 1.6 & 1.6 & 1.6 & 1.6 & 1.6 & 1.6 \\
ZnO & 2.5 & 2.5 & 2.4 & 2.5 & 2.5 & 2.5 \\
\hline
\end{tabular}

Table 1: Material compositions in parts per hundred rubber (phr).

applying the time-temperature superposition principle, it was possible to plot the storage and loss moduli for a wide range a frequencies that cannot be obtained experimentally at a fixed temperature. The storage modulus $E^{\prime}$ and the loss modulus $E^{\prime \prime}$ master curves built for a reference temperature of $0^{\circ} \mathrm{C}$ are shown in Fig. 2. During the master curve building process, we noticed that the Williams-Landel-Ferry equation (Williams et al., 1955) applied well for each material.

In the next section, we use a micromechanics modeling approach in order to reproduce the linear viscoelasticity of the filled rubbers that are heterogeneous materials and are recognized as composites.

\section{Modeling}

\subsection{Micromechanics modeling}

By accounting for the microstructure (amount of fillers, geometry, size...) and for the mechanical behavior of each constitutive phase, micromechanics modeling intends to predict the mechanical behavior of composite materials (Nemat-Nasser and Hori, 1999). In the case of filled rubbers, the material may be described to a first approximation as a rubber matrix reinforced by rigid spherical fillers. This description may seem somewhat simplistic but we will see in the following that it is relevant for the prediction of the linear viscoelasticity of our materials. The major interest of such a representation is that it provides analytical solutions for a large number of micromechanics models for elastic composites. Therefore, by merely applying the elasticviscoelastic correspondence principle stated by Hashin (1970), these solutions extend to linear viscoelasticity when replacing the elastic parameters by the 


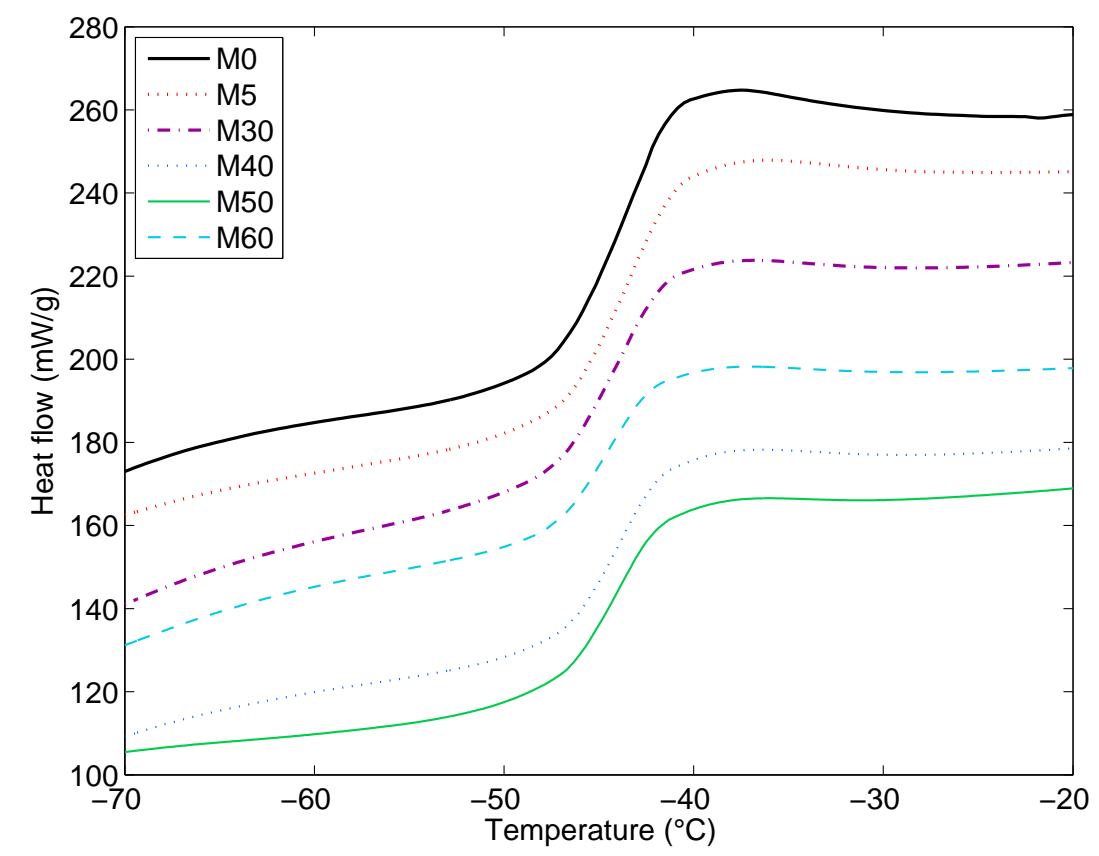

Figure 1: DSC curves for the six SBR materials.
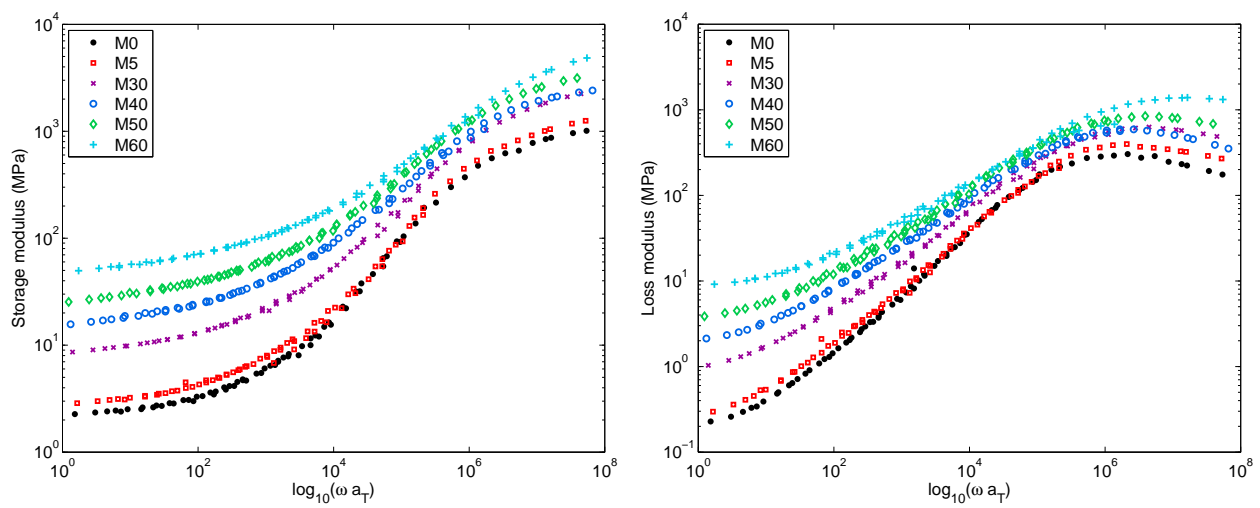

Figure 2: Uniaxial tension storage modulus (Left) and loss modulus (Right) master curves for materials M0, M5, M30, M40, M50 and M60 obtained by time-temperature superposition at a reference temperature of $0^{\circ} \mathrm{C}$. 
(a)

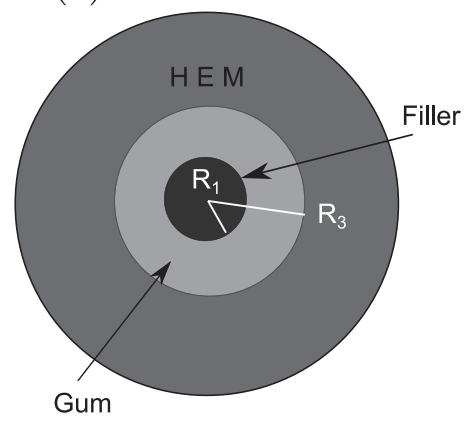

(b)

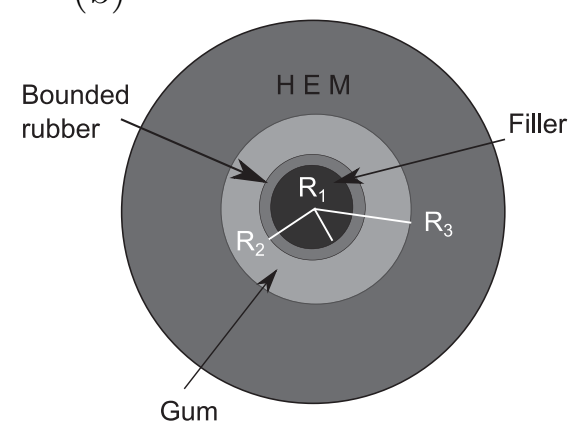

Figure 3: 3-phase (a) and 4-phase (b) model representations.

suitable complex viscoelastic parameters. One may consider such models as the 3-phase model (or generalized self-consistent model, see equations in Appendix 1) introduced by Christensen and Lo (1979, 1986), for which the fillers surrounded by rubber gum are embedded in the homogeneous equivalent medium, which is the composite medium, or as the 4-phase model accounting for the existence of a filler-rubber interphase (detailed equations are given in Appendix 2). In the latter model introduced by Maurer (1990) and generalized by Hervé and Zaoui (1993), the filler is assumed coated by an interphase that consists of a rubber layer bounded to the filler. The filler+interphase sphere is surrounded by rubber which behaves as the bulk unfilled rubber, and this layered sphere is assumed to be embedded in the homogeneous equivalent medium. Representations of the 3-phase and the 4-phase models are shown in Fig. 3.

Each micromechanics model provides access to a theoretical estimate of the complex Young modulus $E^{*}=E^{\prime}+i E^{\prime \prime}$ whose real and imaginary parts can be compared to the experimental data plotted in Fig. 2. A satisfactory comparison between the theoretical estimates and the experimental measures provides solid ground for a realistic account of the microstructure and of the phase behaviors and interactions. Inputs for these models are the volume fraction and the behavior of each constitutive phase. The latter may be characterized by the Young modulus and the bulk modulus for instance, since the fillers, the rubber gum and the interphase are assumed isotropic. The complex Young modulus $E^{*}$ of the rubber matrix (material M0) is provided by experimental measures plotted in Fig. 2. In order to use a smooth representation of the storage modulus and the loss modulus of material M0 in 


\begin{tabular}{lcccc}
\hline$\tau_{i}(\mathrm{~s})$ & $1.852 \times 10^{-8}$ & $8.993 \times 10^{-8}$ & $4.368 \times 10^{-7}$ & $2.121 \times 10^{-6}$ \\
$E_{i}(\mathrm{MPa})$ & 320.3 & 293.7 & 235.3 & 86.88 \\
\hline$\tau_{i}(\mathrm{~s})$ & $1.030 \times 10^{-5}$ & $5.004 \times 10^{-5}$ & $2.431 \times 10^{-4}$ & $1.181 \times 10^{-3}$ \\
$E_{i}(\mathrm{MPa})$ & 18.79 & 4.283 & 2.488 & 0.8908 \\
\hline$\tau_{i}(\mathrm{~s})$ & $5.734 \times 10^{-3}$ & $2.785 \times 10^{-2}$ & 0.1353 & 0.6570 \\
$E_{i}(\mathrm{MPa})$ & 0.3943 & 0.2369 & $0.8696 \times 10^{-2}$ & $0.2707 \times 10^{-3}$ \\
\hline
\end{tabular}

Table 2: Generalized Maxwell model parameters fitting the linear viscoelasticity of material M0 (Fig. 4).

our computations, we applied a generalized Maxwell model with one elastic branch in parallel with $n$ Maxwell assemblies, which may be written as:

$$
\left\{\begin{array}{l}
E^{\prime}(\omega)=E_{e}+E_{i} \sum_{i=1}^{n} \frac{\left(2 \omega \tau_{i}\right)^{2}}{1+\left(2 \omega \tau_{i}\right)^{2}} \\
E^{\prime \prime}(\omega)=E_{i} \sum_{i=1}^{n} \frac{2 \omega \tau_{i}}{1+\left(2 \omega \tau_{i}\right)^{2}}
\end{array}\right.
$$

and fits material M0 experimental data (Fig. 4) with $n=12$. The $\left(\tau_{i}, E_{i}\right)$ pairs used to obtain Fig. 4 with $E_{e}=2.3 \mathrm{MPa}$ are reported in Table 2.

The linear viscoelasticity of the bulk modulus is more difficult to reach and is not necessarily required. Actually, when passing the glass transition, the bulk modulus changes by a factor of two approximately while the Young modulus changes by three orders of magnitude. For example, the bulk modulus of a SBR gum was measured to vary from $2 \mathrm{GPa}$ to $3.5 \mathrm{GPa}$ (Wada et al., 1960) when passing the glass transition. Therefore, we assumed the bulk modulus constant and equal to $3.5 \mathrm{GPa}$ and we noted that the results presented in what follows were not modified if assuming the bulk modulus constant and equal to $2 \mathrm{GPa}$. The fillers are assumed as elastic quasi-incompressible (Poisson's ratio close to 0.5) with a Young modulus of $70 \mathrm{GPa}$. During model computations, we noticed that the exact Young modulus value of the fillers had little impact on the results as long as they were assumed significantly stiffer than the gum, which is realistic. When considering the 4-phase model, the behavior and the volume fraction of the interphase are unknown and are to be estimated during computations. 

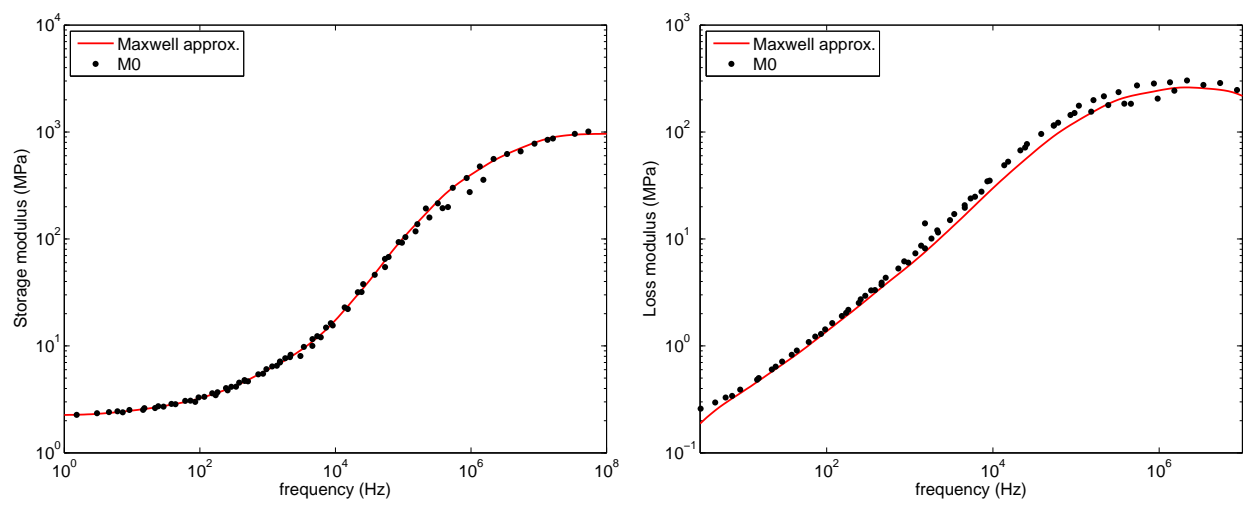

Figure 4: Representation of the storage Young modulus and the loss Young modulus of the unfilled rubber gum (material M0) with a generalized Maxwell model.

\subsection{Effective amount of fillers}

Our initial description of the microstructure consists of spherical fillers surrounded with rubber gum and embedded in the homogeneous medium without accounting for a possible interphase (Fig. 3a). If such an interphase exists, it consists of bounded rubber with reduced mobility. Therefore, the impact of the interphase should not read on the mechanical behavior of the composites when the gum is in a glassy state, and particles surrounded by matrix embedded in the homogeneous medium (3-phase model) may depict the composite microstructures. With this material description, and considering the glassy state, one may predict the effective amount of fillers in each material. Actually, in carbon-black filled rubbers, the volume fraction of the reinforcing rigid phase is significantly underestimated when accounting for the filler volume fraction only. This is due to the presence of rubber gum trapped in between fillers. This occluded gum acts as rigid and therefore enhances the volume fraction of active fillers. Medalia (1972) proposed an estimate of the effective volume fraction of filler calculated from the adsorption of dibutyl-phtalate:

$$
f_{\text {Med }}=\left(0.842+7.325 \times 10^{-3} D B P\right) f
$$

where $f$ is the actual volume fraction of filler reported in section 2.1 and $D B P$ denotes the amount of dibutyl-phtalate adsorbed, in $\mathrm{ml} / 100 \mathrm{~g}$. The coefficients in this equation are based on a phenomenological fit of experimental data depending on the filler surface and how aggregates form. Therefore, 
it does not necessarily strictly apply to our materials but provides a first estimate of the effective volume fraction. Table 3 shows a comparison between the volume fractions of fillers $f$ and the effective volume fractions of fillers $f_{M e d}$ computed from Eq. 2 and from the $D B P$ values measured on our materials, as reported in 2.1. One may notice that the amount of trapped gum contributing to the rigid phase is relatively significant. Another way to assess the amount of effective rigid phase for each material is proposed here: we apply the 3-phase model (Fig. 3a) with the volume fraction of rigid phase being unknown when predicting the composite behavior in the glassy state. A comparison of the model with the experimental data is shown in Fig. 5. The computed effective volume fractions denoted $f_{\text {eff }}$ are listed in Table 3, and one notes that the effective volume fractions provided by the 3 -phase model are larger than the values given by Eq. 2. Nonetheless they remain close except for material M30 for which we noticed a significantly higher reinforcement. For material M60, it has not been possible to compute a value of $f_{\text {eff }}$ that would provide a good comparison between the model and the experimental data. This could be explained by the large amount of fillers present in this material, which may favor filler percolation. In such a case, the simple 3-phase representation fails to capture the microstructure.

It may be observed in Fig. 5 that the 3-phase model is capable to reproduce the composite behaviors at high frequencies only. The glassy state and part of the glass transition frequency range is covered by the model but the other part and the rubbery state are clearly underestimated. This result is in contradiction with Raos (2003), who shows that the 3-phase model can be used to reproduce the rubber elasticity of filled rubbers, but our result is consistent with the effort made by Albérola and co-workers (Albérola et al., 1997, 2001; Mélé et al., 2001) to reproduce the linear viscoelasticity from the rubbery state to the glassy state. This result favors the idea of the existence of a layer of bounded rubber with reduced mobility at the filler-rubber interphase that reinforces the material when the SBR matrix is in the rubbery state. The 4-phase model allows us to test this idea.

\subsection{Account for a filler-matrix interphase}

Berriot et al. (2002b) showed experimental evidences of the existence of a layer of rubber at the filler-gum interphase with reduced mobility in model silica-filled elastomers. This means that when the matrix is in the rubbery state, a filler coating layer of rubber with reduced mobility and enhanced physical crosslinks (Li et al., 2008) strengthens the material. In the glassy 


\begin{tabular}{lcccccc}
\hline Material & M0 & M5 & M30 & M40 & M50 & M60 \\
$f$ & 0 & 2.4 & 13.0 & 16.7 & 20.0 & 23.0 \\
$f_{\text {Med }}$ & 0 & 4.2 & 22.8 & 29.2 & 35.0 & 40.3 \\
$f_{\text {eff }}$ & 0 & 5.0 & 28.0 & 31.0 & 37.0 & - \\
\hline
\end{tabular}

Table 3: Volume fraction and effective volume fraction of fillers in percent.

state, the layer of bounded rubber at the filler-rubber interface behaves like the rubber matrix. Due to the restricted chain mobility within this layer, its stiffness in the rubbery state is probably significantly higher than the rubber matrix stiffness. In order to simply build the interphase viscoelastic behavior, we propose to define it as:

$$
E_{i}^{*}(\omega)=E_{m g}^{*}+x\left(E_{m}^{*}(\omega)-E_{m g}^{*}\right)
$$

where $\omega$ is the frequency, $E_{m}^{*}(\omega)$ the complex Young modulus of the unfilled bulk rubber, $E_{m g}^{*}$ its value in the glassy state and $x \in[0,1]$ is a constant parameter that characterizes the interphase stiffness. Remind that in the glassy state, the material behavior is elastic and satisfies to $E_{m g}^{*}=E_{m g}^{\prime}$, since $E^{\prime \prime}=0$ for an elastic behavior. One may note that the interphase behaves as the rubber matrix when $x=1$, and that the interphase behavior is elastic and coincides with the rubber matrix in the glassy state when $x=0$. The bulk modulus of the interphase is assumed constant and equal to the rubber matrix bulk modulus, since one does not expect a significant change in this modulus with a reduced chain mobility (Diani et al., 2008). In order to run the 4-phase model, two unknowns remain: the interphase volume fraction $f_{i}$ and its behavior, which is now characterized by the $x$ parameter. While the interphase volume fraction is expected to increase with the amount of fillers, it seems reasonable to assume that its behavior does not depend on the filler amount, which means that the same value of $x$ stands for the four materials. The model was applied to the four composite materials M5, M30, M40 and M50 using the effective volume fractions of fillers $f_{\text {eff }}$ displayed in Table 3 , with $x$ and $f_{i}$ standing as fitting parameters, but assuming $x$ identical for all materials. A comparison between the model and the experimental data is shown in Fig. 6. One notes the ability of the 4phase model to reproduce the viscoelasticity of the four composites over the entire frequency range. The interphase viscoelastic behavior providing such interesting results is independent of $f_{\text {eff }}$ and was obtained by taking $x=0.95$ 


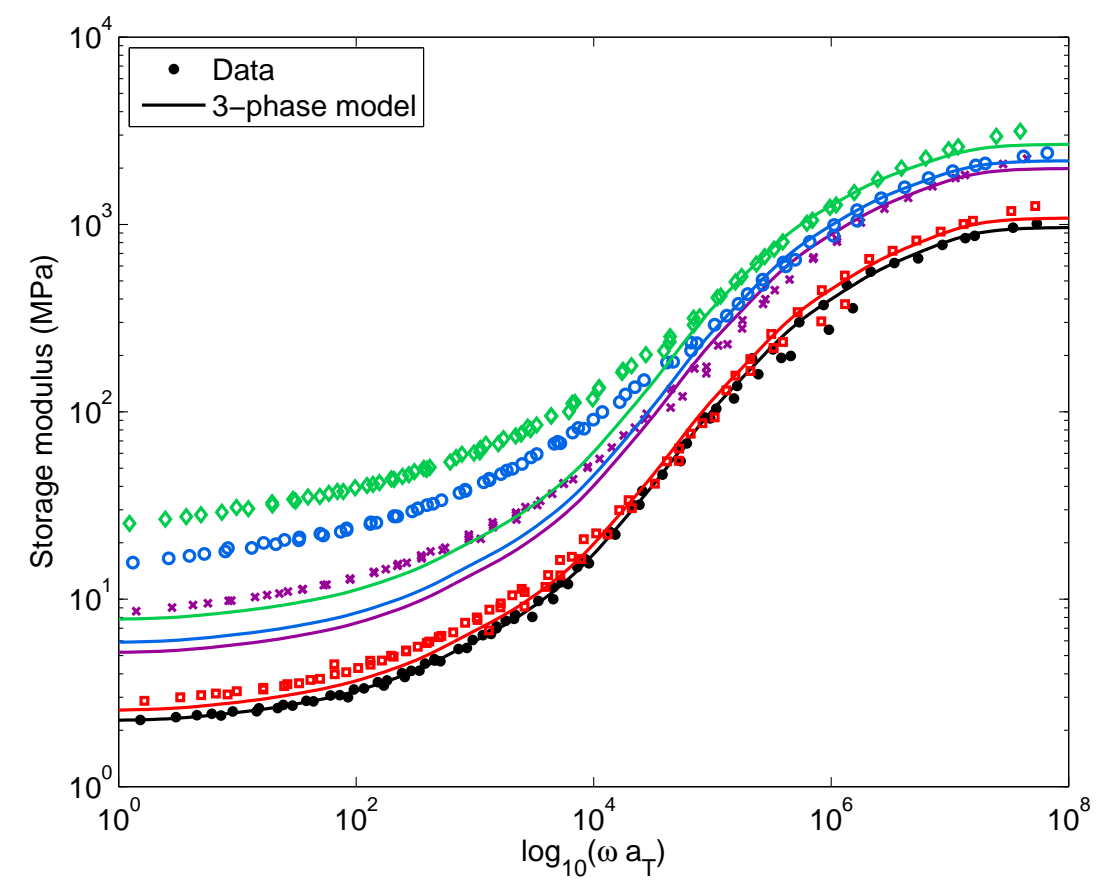

Figure 5: Estimate of the effective amount of fillers by comparison of the material behavior and the theoretical behavior provided by the 3-phase model. The experimental data and the model estimates are ordered from bottom to top according to the amount of fillers from $0,5,30,40$, and $50 \mathrm{phr}$.

in Eq. 3. Fig. 7 shows a comparison between the viscoelastic behaviors of the interphase and of the unfilled bulk rubber. At this point, let us note that it is remarkable to have obtained a value of $x$ providing a good representation of the viscoelasticity for the four composites simultaneously. It is to be noticed that such satisfactory results could not be obtained for $x \leq 0.92$ or $x \geq 0.98$.

The values of the interphase volume fraction $f_{i}$ delivered by the 4-phase model are listed in Table 4. One may note that the interphase volume fraction seems to reach a threshold for M40 and M50. The values reported in Table 4 may seem large and the next step for further discussion on the interest of the 4-phase model for predicting the linear viscoelasticity of filled rubbers could be to compare our results to experimental measures of the volume fraction of bounded rubber. The values of $f_{i}$ and $f_{\text {eff }}$ given in Table 4 and Table 3 can be used to assess the interphase thickness as $\left(\left(f_{i} / f_{\text {eff }}+1\right)^{1 / 3}-1\right) D / 2$. This leads to an average thickness of $4.6 \pm 0.8 \mathrm{~nm}$ for fillers of diameter 

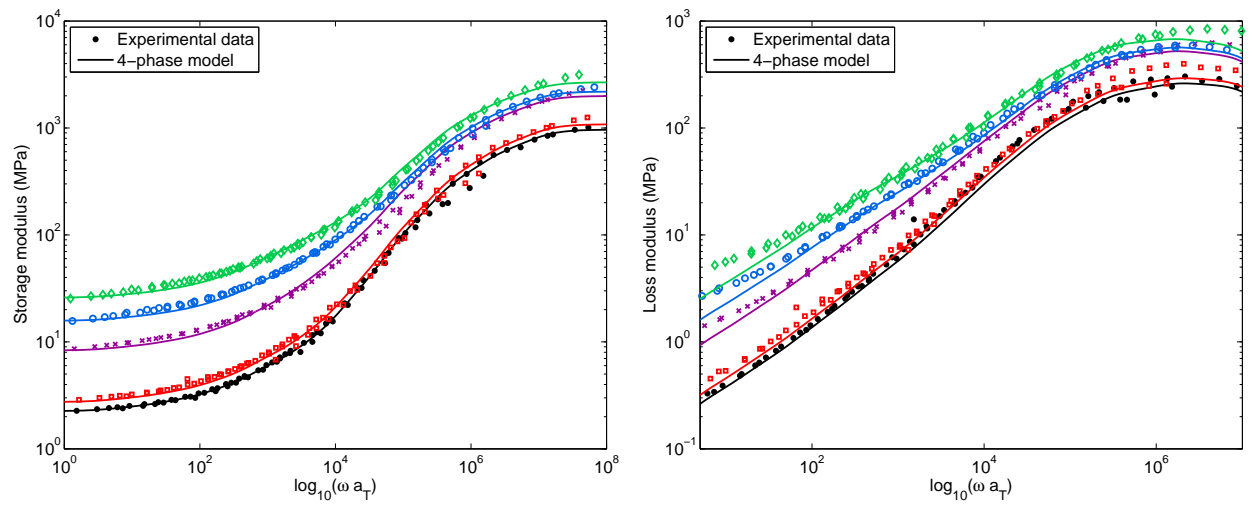

Figure 6: Estimate of the storage modulus and loss modulus of the filled SBRs provided by the 4 -phase model which accounts for the existence of an interphase gum layer with reduced mobility.

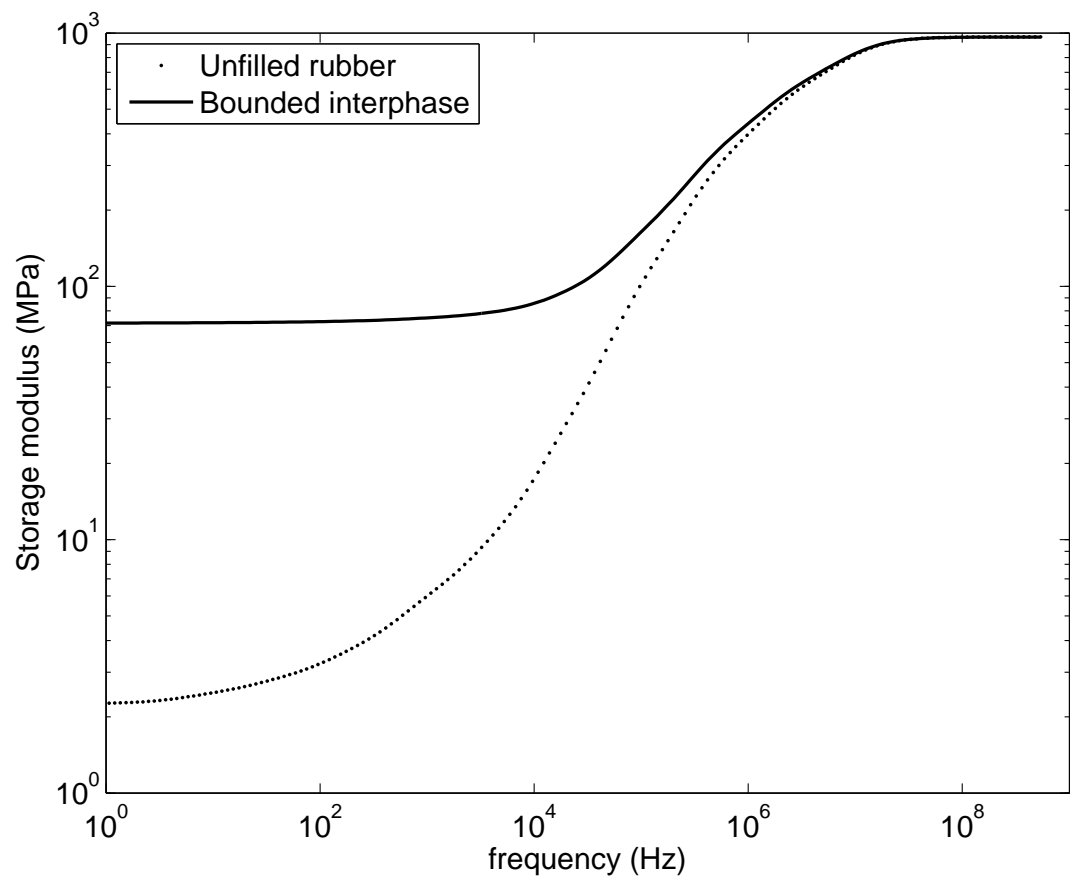

Figure 7: Viscoelastic behavior of the unfilled rubber and of the bounded rubber interphase used as inputs in the 4-phase model for predictions presented in Fig. 6. 


\begin{tabular}{lccccc}
\hline Material & M0 & M5 & M30 & M40 & M50 \\
$f_{i}$ & 0 & 3 & 11 & 19 & 19 \\
\hline
\end{tabular}

Table 4: Volume fraction of interphase in percent.

$D=60 \mathrm{~nm}$, which is realistic in regard of the literature (Berriot et al., 2002b) but remains to be validated on our systems.

As a consequence, the micromechanical modeling could reproduce accurately the linear viscoelasticity of the SBR gum filled with nano-size carbonblack fillers up to $50 \mathrm{phr}$. It supported the existence of a filler-rubber interphase, which is shown to modify significantly the viscoelasticity of the composites in the rubbery state and through the glass transition. The interphase behavior was assessed by the modeling and was shown to be significantly stiffer than the unfilled bulk rubber but still dependent on the frequency and therefore on the temperature due to the time-temperature equivalence shown by the composites.

\section{Conclusion}

A series of carbon-black filled styrene butadiene rubbers were manufactured with the same rubber gum mixed with the same carbon-black (N347), which amount was varied from 5 to $60 \mathrm{phr}$. The materials were tested in DMA to characterize their viscoelasticities.

A 3-phase model was used to assess the effective amount of fillers in each composite. The model is based on a simple representation of the composite as rigid spherical particles surrounded by rubber gum and embedded in the homogeneous equivalent medium. It was shown that the effective amount of reinforcement was significantly larger than the volume of carbon-black added during the material process. It was also shown that this amount was of the order of the effective filler amounts provided by Medalia's equation that accounts for the presence of occluded rubber in the rigid phase. The 3 -phase model also revealed that the 60 phr reinforced material could not be represented by spherical particles in a matrix, setting a limit to the proposed micromechanics representation of our carbon-black filled rubbers.

While the 3-phase model provided satisfying results when the rubber is in glassy state, it was found to largely underestimate the stiffness of the filled rubbers in the rubbery state, favoring the existence of a rubber layer 
with reduced mobility at the filler-rubber interface. Therefore, a 4-phase model accounting for the presence of such a rubber-filler interphase was used and proved to accurately reproduce the experimental data. This provided estimates for the interphase behavior and its volume fraction according to the amount of fillers that remains to be compared to experimental measures.

\section{Acknowledgement}

This work was supported by the French Agence Nationale de la Recherche through project AMUFISE grant MATETPRO 08-320101.

\section{References}

Albérola, N.D., Mélé, P., 1997. Interface and mechanical coupling effects in model particulate composites. Polym. Eng. Sci., 37, 1712-1721.

Albérola, N.D., Benzarti, K., Bas, C., Bomal, Y., 2001. Interface effects in elastomers reinforced by modified precipitated silica. Polym Composites, 22, 312-325.

Aldraihem, O.J., 2011. Micromechanics modeling of viscoelastic properties of hybrid composites with shunted and arbitrarily oriented piezoelectric inclusions. Mech. Mater., 43, 740-753.

Berriot, J., Lequeux, F., Monner, L., Montes, H., Long, D., Sotta, P., 2002a. Filler-elastomer interaction in model filled rubbers, a $1 \mathrm{H}$ NMR study. J. Non-Crys. Solids, 310, 719-724.

Berriot, J., Montes, H., Lequeux, F., Long, D., Sotta, P., 2002b. Evidence of the shift of the glass transition near the particles in silica-filled elastomers. Macromolecules, 35, 9756-9762.

Christensen, R.M., Lo K.H., 1979. Solution for effective shear properties in three phase sphere and cylinder models. J. Mech. Phys. Solids, 27, 315330.

Christensen, R.M., Lo, K.H., 1986. Solution for effective shear properties in three phase sphere and cylinder models. J. Mech. Phys. Solids, 34, 639. 
Diani, J., Gilormini, P., Fayolle, B., 2008. Study on the temperature dependence of the bulk modulus of polyisoprene by molecular dynamics simulations. Mol. Simul ., 34, 1143-1148.

Hashin, Z., 1970. Complex moduli of viscoelastic composites. I. General theory and application to particulate composites. Int. J. Solids Struct., 6, 539-52.

Heinrich, G., Klüppel, M., 2002. Recent advances in the theory of filler networking in elastomers. Adv. Polym. Sci., 160, 1-44.

Hervé. E., Zaoui., A., 1993. n-layered inclusion-based micromechanical modeling. Int. J. Engng. Sci., 31, 1-10.

Léopoldès, J., Barrès, C., Leblanc, J.L., Georget, P., 2004. Influence of filler-rubber interactions on the viscoelastic properties of carbon-blackfilled rubber compounds. J. Appl. Polym. Sci., 91, 577-588.

Li, Z.H., Zhang, J., Chen, S.J., 2008. Effects of carbon blacks with various structures on vulcanization and reinforcement of filled etylenepropylene-diene rubber. Express Polym. Lett., 2, 695-704.

Maurer, F.H.J., 1990. An interlayer model to describe the physical properties of particulate composites. In: Ishida H, editor, Controlled interphases in composite materials. Elsevier Science Publishing Co, pp. 491504.

Medalia, A.I., 1972. Effective degree of immobilization of rubber occluded within carbon black aggregates. Rubber Chem. Technol., 45, 1171-1194.

Mélé, P., Marceau, S., Brown, D., de Puydt, Y., Albérola, N.D., 2002.

Reinforcement effects in fractal-structure-filled rubber. Polymer, 43, $5577-5586$.

Nemat-nasser, S., Hori, M., 1999. Micromechanics: overall properties of heterogeneous materials, second revision edition. Elsevier, Amsterdam.

Raos, G., 2003. Application of the Christensen-Lo model to the reinforcement of elastomers by fractal fillers. Macromol. Theory Simul., 12, 1723. 
Seidel, G.D., Lagoudas, D.C., 2005. Micromechanical analysis of the effective elastic properties of carbon nanotube reinforced composites. Mech. Mater., 38, 884-907.

Williams, M.L., Landel, R.F., Ferry, J.D., 1955. The temperature dependence of the relaxation mechanisms in amorphous polymers and other glass forming liquids. J. Amer. Chem. Soc., 77, 3701-3707.

Wada, Y., Hirose, H., Umebayashi, H., Otomo M., 1960. Volume viscoelasticity of polymers and other highly dissipative materials. J. Phys. Soc. Jap., 15, 2324-2334.

\section{Appendix 1: the 3-phase model}

The 3-phase model (Christensen and Lo, 1979, 1986) involves the equations given below when applied to isotropic elastic constituents defined by the following volume fractions, bulk moduli, shear moduli, and Poisson's ratios: $f_{g}, K_{g}, G_{g}$, and $\nu_{g}$ for the gum phase, $f_{p}, K_{p}, G_{p}$, and $\nu_{p}$ for the filler (particles) phase, with $f_{g}+f_{p}=1$. The bulk modulus $K$ of the composite is

$$
K=K_{g}+\frac{f_{p}}{\frac{1}{K_{p}-K_{g}}+\frac{3 f_{g}}{3 K_{g}+4 G_{g}}} .
$$

The shear modulus $G$ of the composite is the positive root of

$$
A G^{2}-B G_{g} G+C G_{g}^{2}=0
$$

where

$$
\begin{gathered}
A=8\left(5 \nu_{g}-4\right) g e_{1} f_{p}^{10 / 3}+D+50\left(8 \nu_{g}^{2}-12 \nu_{g}+7\right) g e_{2} f_{p}+4\left(10 \nu_{g}-7\right) e_{2} e_{3} \\
B=4\left(5 \nu_{g}-1\right) g e_{1} f_{p}^{10 / 3}+2 D-150\left(\nu_{g}-3\right) \nu_{g} g e_{2} f_{p}+3\left(15 \nu_{g}-7\right) e_{2} e_{3} \\
C=-4\left(5 \nu_{g}-7\right) g e_{1} f_{p}^{10 / 3}+D-25\left(\nu_{g}^{2}-7\right) g e_{2} f_{p}+\left(5 \nu_{g}+7\right) e_{2} e_{3}
\end{gathered}
$$

with

$$
\begin{gathered}
g=G_{p} / G_{g}-1, \quad D=2\left[63 g e_{2}+2 e_{1} e_{3}\right] f_{p}^{7 / 3}-252 g e_{2} f_{p}^{5 / 3}, \\
e_{1}=\left(49+35 \nu_{p}-70 \nu_{g}-50 \nu_{p} \nu_{g}\right) g+105\left(\nu_{p}-\nu_{g}\right), \\
e_{2}=\left(7+5 \nu_{p}\right) g+35\left(1-\nu_{p}\right), \quad e_{3}=2\left(4-5 \nu_{g}\right) g+15\left(1-\nu_{g}\right) .
\end{gathered}
$$




\section{Appendix 2: the 4-phase model}

The 4-phase model (Maurer, 1990) involves the following equations when applied to isotropic elastic constituents defined like in Appendix 1 plus $f_{i}$, $K_{i}, G_{i}$, and $\nu_{i}$ for the interphase, with $f_{g}+f_{p}+f_{i}=1$. The 3 -phase model is recovered when $f_{i}=0$, among other special cases. The bulk modulus $K$ of the composite is

$$
K=K_{g}+\frac{f_{p}+f_{i}}{\frac{1}{K_{e}-K_{g}}+\frac{3 f_{g}}{3 K_{g}+4 G_{g}}}
$$

where

$$
K_{e}=K_{i}+\frac{f_{p}}{\frac{f_{p}+f_{i}}{K_{p}-K_{i}}+\frac{3 f_{i}}{3 K_{i}+4 G_{i}}} .
$$

The shear modulus $G$ of the composite is the positive root of

$$
40 \operatorname{det}[X] G^{2}+(2 \operatorname{det}[Y]+8 \operatorname{det}[Z]) G_{g} G-5 \operatorname{det}[T] G_{g}^{2}=0
$$

where the determinants of four $10 \times 10$ matrices $[X],[Y],[Z]$, and $[T]$ are involved. The only non-zero elements of $[X]$ are

$$
\begin{gathered}
X(1,1)=X(2,1)=G_{p} / G_{i}, \quad X(1,2)=-f_{2} G_{p} /\left(2 G_{i}\right), \\
X(1,3)=X(2,3)=X(5,7)=X(6,7)=-1, \quad X(1,4)=4 / f_{5}, \\
X(1,5)=f_{2} / 2, \quad X(1,6)=-a_{i} / f_{p}, \quad X(2,2)=b_{p} f_{2} G_{p} / G_{i}, \\
X(2,4)=-8 /\left(3 f_{5}\right), \quad X(2,5)=-b_{i} f_{2}, \quad X(2,6)=-c_{i} / f_{p}, \\
X(3,1)=-X(3,3)=f_{1}, \quad X(3,2)=-X(3,5)=f_{p}, \quad X(3,4)=-1 / f_{4}, \\
X(3,6)=-1 / f_{2}, \quad X(4,1)=-X(4,3)=f_{1} / 2, \quad X(4,2)=d_{p} f_{p}, \\
X(4,4)=1 /\left(3 f_{4}\right), \quad X(4,5)=-d_{i} f_{p}, \quad X(4,6)=-e_{i} / f_{2}, \\
X(5,3)=X(6,3)=G_{i} / G_{g}, \quad X(5,4)=-4 G_{i} /\left(f_{5}^{\prime} G_{g}\right), \\
X(5,5)=-f_{2}^{\prime} G_{i} /\left(2 G_{g}\right), \quad X(5,6)=a_{i} G_{i} /\left(f^{\prime} G_{g}\right), \quad X(5,8)=4 / f_{5}^{\prime}, \\
X(5,9)=f_{2}^{\prime} / 2, \quad X(5,10)=-a_{g} / f^{\prime}, \quad X(6,4)=8 G_{i} /\left(3 f_{5}^{\prime} G_{g}\right), \\
X(6,5)=b_{i} f_{2}^{\prime} G_{i} / G_{g}, \quad X(6,6)=c_{i} G_{i} /\left(f^{\prime} G_{g}\right), \quad X(6,8)=-8 /\left(3 f_{5}^{\prime}\right), \\
X(6,9)=-b_{g} f_{2}^{\prime}, \quad X(6,10)=-c_{g} / f^{\prime}, \quad X(7,3)=-X(7,7)=f_{1}^{\prime}, \\
X(7,4)=-X(7,8)=1 / f_{4}^{\prime}, \quad X(7,5)=-X(7,9)=f^{\prime}, \\
X(7,6)=-X(7,10)=1 / f_{2}^{\prime}, \quad X(8,3)=-X(8,7)=f_{1}^{\prime} / 2, \\
X(8,4)=-X(8,8)=-1 /\left(3 f_{4}^{\prime}\right), \quad X(8,5)=d_{i} f^{\prime}, \quad X(8,6)=e_{i} / f_{2}^{\prime},
\end{gathered}
$$




$$
\begin{gathered}
X(8,9)=-d_{g} f^{\prime}, \quad X(8,10)=-e_{g} / f_{2}^{\prime}, \quad X(9,7)=5 / 2, \\
X(9,9)=1+3 d_{g}, \quad X(9,10)=1+3 e_{g}, \quad X(10,7)=1 / 2, \\
X(10,8)=-1 / 3, \quad X(10,9)=d_{g}, \quad X(10,10)=e_{g}
\end{gathered}
$$

where

$$
\begin{gathered}
f_{1}=f_{p}^{1 / 3}, \quad f_{2}=f_{p}^{2 / 3}, \quad f_{4}=f_{p}^{4 / 3}, \quad f_{5}=f_{p}^{5 / 3}, \\
f_{1}^{\prime}=\left(f^{\prime}\right)^{1 / 3}, \quad f_{2}^{\prime}=\left(f^{\prime}\right)^{2 / 3}, \quad f_{4}^{\prime}=\left(f^{\prime}\right)^{4 / 3}, \quad f_{5}^{\prime}=\left(f^{\prime}\right)^{5 / 3}
\end{gathered}
$$

with $f^{\prime}=f_{p}+f_{i}$ and

$$
\begin{gathered}
a_{g}=-2\left(5-\nu_{g}\right) /\left(5-4 \nu_{g}\right), \quad a_{i}=-2\left(5-\nu_{i}\right) /\left(5-4 \nu_{i}\right), \\
b_{g}=\left(7+2 \nu_{g}\right) /\left(6 \nu_{g}\right), \quad b_{p}=\left(7+2 \nu_{p}\right) /\left(6 \nu_{p}\right), \\
b_{i}=\left(7+2 \nu_{i}\right) /\left(6 \nu_{i}\right), \quad c_{g}=2\left(1+\nu_{g}\right) /\left(5-4 \nu_{g}\right), \\
c_{i}=2\left(1+\nu_{i}\right) /\left(5-4 \nu_{i}\right), \quad d_{g}=\left(7-4 \nu_{g}\right) /\left(12 \nu_{g}\right), \\
d_{p}=\left(7-4 \nu_{p}\right) /\left(12 \nu_{p}\right), \quad d_{i}=\left(7-4 \nu_{i}\right) /\left(12 \nu_{i}\right), \\
e_{g}=\left(1-2 \nu_{g}\right) /\left(5-4 \nu_{g}\right), \quad e_{i}=\left(1-2 \nu_{i}\right) /\left(5-4 \nu_{i}\right) .
\end{gathered}
$$

The only elements of $[Y]$ that differ from $[X]$ are

$$
\begin{gathered}
Y(9,7)=Y(10,8)=0, \quad Y(9,8)=-20 / 3, \\
Y(9,9)=-1 / 2-b_{g}, \quad Y(9,10)=a_{g}-c_{g}, \quad Y(10,7)=5 / 2, \\
Y(10,9)=1+3 d_{g}, \quad Y(10,10)=1+3 e_{g}
\end{gathered}
$$

whereas the only elements of $[Z]$ that differ from $[Y]$ are

$$
\begin{gathered}
Z(9,7)=5 / 2, \quad Z(9,8)=0, \quad Z(9,9)=-1 / 2+3 b_{g} / 2, \\
Z(9,10)=a_{g}+3 c_{g} / 2, \quad Z(10,7)=0, \quad Z(10,8)=5 / 3, \\
Z(10,9)=1-2 d_{g}, \quad Z(10,10)=1-2 e_{g}
\end{gathered}
$$

and the only elements of $[T]$ that differ from $[Z]$ are

$$
T(10,7)=1, \quad T(10,8)=8 / 3, \quad T(10,9)=b_{g}, \quad T(10,10)=c_{g} .
$$

